\title{
Fast Radio Burst Distributions Consistent with the First CHIME/FRB Catalog
}

\author{
Da-Chun Qiang * Shu-Ling Li, and Hao Wei \\ School of Physics, Beijing Institute of Technology, Beijing 100081, China
}

\begin{abstract}
Currently, fast radio bursts (FRBs) have become a very active field in astronomy and cosmology. However, the origin of FRBs is still unknown to date. The studies on the intrinsic FRB distributions might help us to reveal the possible origins of FRBs, and improve the simulations for FRB cosmology. Recently, the first CHIME/FRB catalog of 536 events was released. Such a large uniform sample of FRBs detected by a single telescope is very valuable to test the FRB distributions. Later, it has been claimed that the FRB distribution model tracking the cosmic star formation history (SFH) was rejected by the first CHIME/FRB catalog. In the present work, we consider some empirical FRB distribution models, and find that many of them can be fully consistent with the CHIME/FRB observational data for some suitable model parameters. Notice that a suppressed evolution with respect to SFH is commonly found for FRBs. In particular, we independently confirm that the FRB distribution model tracking SFH can be rejected at very high confidence. On the other hand, all the "successful" models effectively require a certain degree of "delay" with respect to SFH. These results might shed light on the origin of FRBs and FRB cosmology.
\end{abstract}

PACS numbers: 98.80.Es, 98.70.Dk, 98.70.-f, 97.10.Bt, 98.80.-k

* email address: 875019424@qq.com

${ }^{\dagger}$ Corresponding author; email address: haowei@bit.edu.cn 


\section{INTRODUCTION}

Fast radio bursts (FRBs) are mysterious transient radio sources of millisecond duration [1 10]. More than a decade passed since their discovery [11, 12], but the origin of FRBs is still unknown to date [6-10]. Many theoretical models have been proposed for the engines of FRBs in the literature (see e.g. [6 [10] for comprehensive reviews and [13] for the up-to-date online catalogue of FRB theories). These theoretical models predicted quite different FRB distributions, especially the intrinsic redshift distribution for FRBs (see e.g. Fig. 1 of [14] for a nice summary). So, the studies on the intrinsic FRB distributions might help us to reveal the possible origins of FRBs.

The large dispersion measures (DMs) of observed FRBs well in excess of the Galactic values suggest that FRBs are at extragalactic/cosmological distances. Thus, it is reasonable to study cosmology and the intergalactic medium (IGM) by using FRBs (see e.g. [15 36]). To this end, a large number of FRBs with identified redshifts is required. However, this is still not available currently. In fact, only around 20 FRB host galaxies and their redshifts were identified to date [37]. Therefore, one has to instead use the simulated FRBs with mock redshifts to study cosmology in the literature (see e.g. [15 36]). In the simulations, one should assume an FRB redshift distribution, and randomly generate mock redshifts for the simulated FRBs by using the assumed redshift distribution. Then, one can study cosmology with this mock sample of simulated FRBs. However, the actual redshift distribution of FRBs is still unknown to date. Thus, various redshift distributions for FRBs have been assumed in the literature. But it was found in e.g. [36] that some biases might rise from these assumed FRB redshift distributions. Therefore, it is also important to seek the realistic redshift distributions from the actual FRB data, which might shed light on the simulations in FRB cosmology.

The important FRB distributions include the intrinsic redshift distribution and the intrinsic energy (or luminosity) distribution. Note that the specific fluence $\left(F_{\nu}\right)$ distribution is a convolution of the redshift and energy distributions. It is commonly believed that the specific fluence distribution is insensitive to these two distributions $[6,9,38-40]$. In the literature, it is usually modeled as a power law $N\left(>F_{\nu}\right) \propto F_{\nu}^{\lambda}$, in particular $\lambda=-3 / 2$ for a non-evolving population in Euclidean space [6, 9, 38- 40]. On the other hand, the isotropic energy $(E)$ distribution can be well constrained by the observations as a power law $d N / d E \propto E^{-\alpha}$ with $1.8 \lesssim \alpha \lesssim 2$ roughly [14, 39, 41 44]. It might has an exponential cutoff [41, 43],

$$
d N / d E \propto\left(E / E_{c}\right)^{-\alpha} \exp \left(-E / E_{c}\right),
$$

but the cutoff energy $E_{c}$ is not well constrained. Although it was suggested to be $E_{c} \sim 3 \times 10^{41} \mathrm{erg}$ in e.g. [38, 43], other values are still acceptable.

To date, the intrinsic FRB redshift distribution is poorly known, mainly due to the deficiency in FRBs with identified redshifts 37]. As a crude rule of thumb, the FRB redshift $z \sim \mathrm{DM} /\left(1000 \mathrm{pc} \mathrm{cm}^{-3}\right)[2]$. The detailed theoretical DM $-z$ relation was given by e.g. [15, 45 47] (see also e.g. [16 [19, 26, 27, 34 36]). It has been confirmed by the recent observations [47, 48]. Thus, one might consider DM as a rough proxy of redshift $z$. In principle, one could constrain the FRB redshift distribution with the observed fluence, energy and DM distributions (see e.g. [14, 38, 49, 50]).

For a long time, it has been speculated that the FRB distribution tracks the cosmic star formation history (SFH) 1-10]. Recently, the landmark Galactic FRB 200428 associated with the young magnetar SGR 1935+2154 [51 54] confirmed that at least some (if not all) FRBs originate from young magnetars. Thus, it is reasonable to expect that the FRB distribution is closely correlated with star-forming activities, as observed for the rapid repeaters [55 [57]. However, the recently discovered repeating FRB 20200120E in a globular cluster of the nearby galaxy M81 [58 60] suggested that at least some FRBs are associated with old stellar populations [38]. It challenges FRB models that invoke young magnetars formed in a core-collapse supernova as powering FRB emission. It was proposed in e.g. [59] that FRB 20200120E is a highly magnetized neutron star formed via either accretion-induced collapse of a white dwarf or merger of compact stars in a binary system. So, a delayed FRB distribution tracking compact binary mergers is also reasonable (see e.g. [14, 38, 61, 62]).

In [14], two types of intrinsic FRB distribution models (tracking SFH and compact binary mergers respectively) were tested by using the observed samples from ASKAP and Parkes telescopes, respectively. Both observational samples have $27 \mathrm{FRB}$ events each. It was found that these distribution models can be consistent with the observational data as of October 2020, while the compact binary merger models 
required the characteristic delay timescales of $2 \sim 3$ Gyr [14]. In [49], another FRB distribution model $d N /(d t d V) \propto(\operatorname{SFH}(z))^{n} /(1+z)$ was tested by using a sample of 24 non-localized plus 7 localized FRBs detected by ASKAP, and 20 FRBs detected by the Parkes multibeam system. It was found that a nonevolving population can be ruled out at $99.9 \%$ C.L. The FRB distribution was consistent with SFH, but FRB progenitors that evolve faster than SFH were also allowed as of 2020 [49]. Note that the $n$-th power SFH model considered in [49] is a phenomenological model which lacks a physical meaning. In [61], it was found that the delayed FRB distribution tracking compact binary mergers with the characteristic delay timescales of a few hundreds Myr can be consistent with 22 Parkes FRBs as of December 2017. In [62], it was found that the FRB distribution tracking SFH can be consistent with 20 Parkes FRBs as of January 2016, and the FRB distribution evolving faster than SFH can be consistent with 23 ASKAP FRBs as of May 2018. But the delayed FRB distribution cannot be consistent with both samples. Note that these works in e.g. [14, 49, 61, 62] used the observational samples consisting of only a few tens of FRB events. Thus, the constraints were fairly loose due to the deficiency in the number of data, and hence almost all FRB distribution models can be consistent with the observational data at that time.

Recently, the first CHIME/FRB catalog of 536 events (including 474 one-off bursts and 62 repeat bursts from 18 repeaters) was released in June 2021 63. Clearly, such a large uniform sample of FRBs detected by a single telescope is very valuable to test the FRB distributions. In [50], the first CHIME/FRB catalog was used to study a variety of FRB distribution models, focusing on DM and scattering distributions. Its main attention was paid to the additional sources of scattering, namely the circumgalactic medium of intervening galaxies and the circumburst medium. On the other hand, in [50] only the intrinsic FRB redshift distributions assuming a constant comoving number density or tracking SFH were considered. In [38], the intrinsic FRB redshift, fluence, energy, $\mathrm{DM}_{\mathrm{E}}$ distributions were confronted with the first CHIME/FRB catalog. Four families of redshift distribution models were considered. The SFH model and the accumulated model were rejected with high significance by the Kolmogorov-Smirnov (KS) test against both the energy and $\mathrm{DM}_{\mathrm{E}}$ distributions inferred from the observational data. The delayed model and the hybrid model were not rejected by the KS test with both the fluence and energy criteria, at the price of a very large lognormal delay with a central value of 10 and $13 \mathrm{Gyr}$, respectively. Note that these $10 \sim 13$ Gyr delays are significantly larger than the one of short gamma-ray bursts $(2 \sim 3$ Gyr $)$ [14, 38], and they are actually comparable with the entire life of our universe $(\sim 13.8 \mathrm{Gyr})[64]$. On the other hand, both the delayed model and the hybrid model were still rejected by the KS test with respect to the $\mathrm{DM}_{\mathrm{E}}$ criterion. It was argued in [38] that the value of $\mathrm{DM}_{\text {host }}$ might partially account for the $\mathrm{DM}_{\mathrm{E}}$ discrepancy.

In the present work, we try to find some empirical FRB distribution models fully consistent with the first CHIME/FRB catalog. We extend the works in [14, 38] and try to make these FRB distribution models can pass all the fluence, energy, $\mathrm{DM}_{\mathrm{E}}$ criteria being not rejected by the KS test, even with the same $\mathrm{DM}_{\text {host }}$ as in [14, 38]. In Sec. II] we briefly describe the methodology following [14, 38], but with some key modifications. In Sec. III, we propose our FRB distribution models, and confront them with the first CHIME/FRB catalog. We find that many of them can be fully consistent with the observational data for some suitable model parameters. In Sec. IV some brief concluding remarks are given.

\section{METHODOLOGY}

At first, we briefly describe the methodology used in the present work, following [14, 38] but with some key modifications making sensible differences. The key idea is to confront the Monte Carlo simulations with the observational data. If the simulations are rejected by the observational data, the assumed FRB distribution models generating these simulations could be ruled out. Otherwise, they survive.

\section{A. Monte Carlo simulations}

As in [14, 38], we perform the Monte Carlo simulations in two steps: generating mock FRBs from the assumed intrinsic FRB distribution models, and then filtering them due to the telescope's sensitivity threshold and instrumental selection effects near the threshold. The filtered FRBs as a mock "observed" sample will be confronted with the observational data. 
First, we generate mock FRBs characterized by the isotropic energy $E$, redshift $z$, specific fluence $F_{\nu}$, and $\mathrm{DM}_{\mathrm{E}}$ (the extragalactic DM). Following [14, 38], we adopt the energy distribution modeled by Eq. (1). In principle, $\alpha$ and $E_{c}$ are both free model parameters as in [14, 38]. To save the computational power and time, it is better to reduce the number of free model parameters. One can see that they take almost the same values for all the four FRB distribution models in [38]. Thus, we fix $\alpha=1.9$ and $\log E_{c}=41$ in the present work, since they hold for four and three models in [38] respectively, where the energy is in units of erg, and "log" gives the logarithm to base 10. On the other hand, it is useless to simulate very low-energy FRBs at cosmological distances, because they cannot be detected by the telescope with a threshold in specific fluence. We find that the lower bound $10^{37} \mathrm{erg}$ is suitable for the CHIME telescope under consideration (actually we have tested other much lower bounds and found almost no difference after filter). Due to the exponential cutoff in Eq. (1), very high-energy FRBs are extremely rare. So, it is enough to randomly sample $E$ from the energy distribution (11) in the range of $10^{37} \sim 10^{44} \mathrm{erg}$.

The observed FRB redshift rate distribution reads [14, 38]

$$
\frac{d N}{d t_{\mathrm{obs}} d z}=\frac{1}{1+z} \cdot \frac{d N}{d t d V} \cdot \frac{d V}{d z}
$$

where we have used $d t / d t_{\mathrm{obs}}=(1+z)^{-1}$ due to the cosmic expansion. The redshift-dependent specific comoving volume in a flat Friedmann-Robertson-Walker (FRW) universe is given by

$$
\frac{d V}{d z}=4 \pi d_{C}^{2} \frac{c}{H(z)}
$$

where the comoving distance $d_{C}=d_{L} /(1+z), d_{L}$ is the luminosity distance at redshift $z, c$ is the speed of light, and $H(z)$ is the Hubble parameter. Throughout this work, we consider the fiducial cosmology, namely the flat $\Lambda$ CDM model, and hence

$$
d_{C}=\frac{d_{L}}{1+z}=c \int_{0}^{z} \frac{d \tilde{z}}{H(\tilde{z})}, \quad H(z)=H_{0} E(z)=H_{0}\left[\Omega_{m}(1+z)^{3}+\left(1-\Omega_{m}\right)\right]^{1 / 2},
$$

where $H_{0}$ is the Hubble constant, and $\Omega_{m}$ is the present fractional density of dust matter (see below for their values). To date, the intrinsic event rate density distribution $d N /(d t d V)$ is poorly known. If the intrinsic FRB redshift distribution tracks SFH, one has $d N /(d t d V) \propto \operatorname{SFH}(z)$. In [14, 38], the analytical three-segment empirical model of [65] was used for $\operatorname{SFH}(z)$ (see Eq. (5) of [14]). However, the analytical two-segment empirical model in the well-known review on SFH [66] (i.e. its Eq. (15)) was used extensively in the literature. In this work, we instead use its updated version given by Eq. (1) of [67], namely

$$
\operatorname{SFH}(z) \propto \frac{(1+z)^{2.6}}{1+((1+z) / 3.2)^{6.2}},
$$

which behaves like $(1+z)^{2.6}$ and $(1+z)^{-3.6}$ at low- and high-redshifts, respectively. In fact, the actual $d N /(d t d V)$ is still unclear to date, and many models have been proposed for it in the literature. Once $d N /(d t d V)$ is given, we can randomly sample redshift $z$ from Eq. (2) for each mock FRB.

As is well known, the observed DM of FRB can be separated into [15-19, 26, 27, 34 36 ]

$$
\mathrm{DM}_{\mathrm{obs}}=\mathrm{DM}_{\mathrm{MW}}+\mathrm{DM}_{\text {halo }}+\mathrm{DM}_{\mathrm{IGM}}+\mathrm{DM}_{\text {host }} /(1+z),
$$

where $\mathrm{DM}_{\mathrm{MW}}, \mathrm{DM}_{\text {halo }}, \mathrm{DM}_{\mathrm{IGM}}$, and $\mathrm{DM}_{\text {host }}$ are the contributions from the Milky Way, the Milky Way halo, the intergalactic medium (IGM), and the host galaxy (including interstellar medium of the host galaxy and the near-source plasma), respectively. It is convenient to introduce the extragalactic DM as

$$
\mathrm{DM}_{\mathrm{E}}=\mathrm{DM}_{\mathrm{obs}}-\mathrm{DM}_{\mathrm{MW}}-\mathrm{DM}_{\text {halo }}=\mathrm{DM}_{\mathrm{IGM}}+\mathrm{DM}_{\text {host }} /(1+z) .
$$

Following [14, 38], here we adopt $\mathrm{DM}_{\text {halo }}=30 \mathrm{pc} \mathrm{cm}^{-3}$ (see e.g. [68, 69]) and $\mathrm{DM}_{\text {host }}=107 \mathrm{pc} \mathrm{cm}^{-3}(\mathrm{see}$ e.g. [70]). For a mock FRB, we can calculate the corresponding $\mathrm{DM}_{\mathrm{E}}=\mathrm{DM}_{\mathrm{IGM}}+\mathrm{DM}_{\text {host }} /(1+z)$ with (see e.g. [15, 16, 26, 27, 34 36])

$$
\mathrm{DM}_{\mathrm{IGM}}=\frac{3 c H_{0} \Omega_{b}}{8 \pi G m_{p}} \int_{0}^{z} \frac{f_{\mathrm{IGM}}(\tilde{z}) f_{e}(\tilde{z})(1+\tilde{z}) d \tilde{z}}{E(\tilde{z})}
$$


where $\Omega_{b}$ is the present fractional density of baryons, $G$ is the gravitational constant, $m_{p}$ is the mass of proton, $E(z) \equiv H(z) / H_{0}$ is the dimensionless Hubble parameter, $f_{e}(z)$ is the ionized electron number fraction per baryon, and $f_{\mathrm{IGM}}(z)$ is the fraction of baryon mass in IGM. The latter two are functions of redshift $z$ in principle [26, 27, 35]. Following [14, 38], we use the fiducial values $f_{e}=7 / 8$ and $f_{\text {IGM }}=0.84$ in this work. Unlike [38] using the cosmological parameters from the Planck 2015 results [71], we adopt $\Omega_{m}=0.3153, \Omega_{b}=0.0493$, and $H_{0}=67.36 \mathrm{~km} / \mathrm{s} / \mathrm{Mpc}$ instead from the Planck 2018 results [64].

Assuming a flat radio spectrum, the "observed" specific fluence for a mock FRB with isotropic energy $E$ and redshift $z$ is given by $[14,72]$

$$
F_{\nu}=\frac{(1+z) E}{4 \pi d_{L}^{2} \nu_{c}},
$$

where $\nu_{c}$ is the central observing frequency, and the luminosity distance $d_{L}$ is given by Eq. (4). Note that the bandwidth $\Delta \nu$ usually takes the place of $\nu_{c}$ in the literature. But it was argued in 72 ] that it is more appropriate to use $\nu_{c}$ instead. To keep pace with [14, 38], we adopt the same form of $F_{\nu}$ in Eq. (9), with $\nu_{c}=600 \mathrm{MHz}$ for CHIME [63].

However, the mock FRBs intrinsically generated above are not the ones "detected" by the telescope, due to the telescope's sensitivity threshold and instrumental selection effects near the threshold. So, the second step is to filter them by using the telescope's sensitivity model, which is difficult to characterize in fact. One might use a sophisticated but fairly complicated model for the selection effects of CHIME as in [50, 63]. Instead, it is convenient to consider the simplified sensitivity model for CHIME as in [38]. Every telescope has a sensitivity threshold. Following [38], it is about 0.3 Jy ms for CHIME, or equivalently $\log F_{\nu \text {, min }}=-0.5$, where the specific fluence is in units of Jy ms. On the other hand, due to the directiondependent sensitivity of the telescope, there is a "gray zone" in the $\log F_{\nu}$ distribution, within which CHIME has not reached full sensitivity to all sources [38]. For this "gray zone", following [38], we adopt $\log F_{\nu, \mathrm{min}}^{\min }=-0.5$ as its minimum threshold specific fluence, while its maximum threshold specific fluence $\log F_{\nu, \text { th }}^{\max }$ should be adjusted to match the "observation". The detection efficiency parameter in the "gray zone" is given by $\eta_{\text {det }}=\mathcal{R}^{3}$, where $\mathcal{R}=\left(\log F_{\nu, \text { th }}-\log F_{\nu, \text { th }}^{\min }\right) /\left(\log F_{\nu, \text { th }}^{\max }-\log F_{\nu, \text { th }}^{\min }\right)$, such that $\eta_{\text {det }} \rightarrow 0$ at $\log F_{\nu, \text { th }}^{\min }$ and $\eta_{\text {det }} \rightarrow 1$ at $\log F_{\nu, \text { th }}^{\max }$ [38]. Outside the "gray zone" $\left(\log F_{\nu, \text { th }}>\log F_{\nu, \text { th }}^{\max }\right), \eta_{\text {det }}=1$. We first screen out the mock FRBs with specific fluences below the threshold $\log F_{\nu \text {, min }}=-0.5$, and then filter the rest with the detection efficiency parameter $\eta_{\text {det }}$. Finally, a mock "observed" sample of FRBs is on hand, which will be confronted with the observational data.

\section{B. CHIME/FRB data}

The data for the first CHIME/FRB catalog in machine-readable format can be downloaded from the public webpage given by 63]. It contains 536 events, including 474 one-off bursts and 62 repeat bursts from 18 repeaters. At first, we exclude the six bursts with zero fluences (detected directly after a system restart) as mentioned at the end of Sec. 3 of [63]. Then, we also exclude the bursts labeled with excluded_flag $=1$ (detected either during pre-commissioning, epochs of low-sensitivity, or on days with software upgrades) as mentioned in the middle of Sec. 6.1 and at the end of Table 2 of [63] (note that they might not be excluded in [38]). Following [38], we only use the first detected burst of each repeating FRB and the non-repeaters. In practice, we identify the non-repeaters labeled with repeater_name $=-9999$ and then only take the ones labeled with sub_num $=0$ (434 bursts in total). We identify the repeaters labeled with repeater_name $\neq-9999$ and only take the ones labeled with sub_num $=0$, and then from them we adopt the first ones in each group with the same repeater_name (18 bursts in total). Finally, we obtain an observational sample of 452 FRBs.

For each burst, its observed $F_{\nu}$ is given by the column labeled with "fluence" in the data table. Its $\mathrm{DM}_{\mathrm{E}}=\mathrm{DM}_{\mathrm{obs}}-\mathrm{DM}_{\mathrm{MW}}-\mathrm{DM}_{\text {halo }}$, in which $\mathrm{DM}_{\mathrm{obs}}$ is given by the column labeled with "bonsai_dm" in the data table, $\mathrm{DM}_{\mathrm{MW}}$ is obtained by using $\mathrm{NE} 2001$ [73, 74], and $\mathrm{DM}_{\text {halo }}=30 \mathrm{pc} \mathrm{cm}^{-3}$ as mentioned above. Then, its inferred redshift $z$ is obtained by numerically solving $\mathrm{DM}_{\mathrm{E}}=\mathrm{DM}_{\mathrm{IGM}}+\mathrm{DM}_{\text {host }} /(1+z)$ with $\mathrm{DM}_{\mathrm{IGM}}$ given by Eq. (8) and $\mathrm{DM}_{\text {host }}=107 \mathrm{pc} \mathrm{cm}^{-3}$ as mentioned above. So, its isotropic energy $E$ can be inferred from Eq. (9) with the observed $F_{\nu}$ and the luminosity distance $d_{L}$ at redshift $z$ given by Eq. (4). Finally, the observational data of 452 FRBs are ready for confrontation with the mock "observed" sample of FRBs. 


\section{Strategy}

As is well known, the Kolmogorov-Smirnov $(\mathrm{KS})$ test is one of the useful tools to compare a sample with a reference probability distribution, or to compare two samples [75]. The KS statistic quantifies the largest distance between the two cumulative distribution functions (CDFs) of the sample and the reference distribution, or two samples. One can perform the KS test by using scipy.stats.kstest in Python [76], which returns the KS statistic and the corresponding p-value. Here we use the p-value $\left(p_{\mathrm{KS}}\right)$ in the two-sample case as in [50, 63], rather than the KS statistic $\left(D_{\mathrm{KS}}\right)$ used in [14, 38]. The null hypothesis (namely two samples are drawn from the same distribution) can be rejected at $90 \%(95 \%)$ confidence if $p_{\mathrm{KS}}<0.1(0.05)$, respectively. Otherwise, two samples can be consistent with each other if $p_{\mathrm{KS}}>0.1$ (or 0.05 ). For two closer samples, $p_{\mathrm{KS}}$ is higher (and $p_{\mathrm{KS}}=1$ for two same samples).

Following [14, 38], the KS test can be used to compare the mock "observed" FRB samples with the CHIME/FRB samples of the specific fluence $F_{\nu}$, the isotropic energy $E$, and the extragalactic dispersion measure $\mathrm{DM}_{\mathrm{E}}$, respectively. As mentioned above, the specific fluence $\left(F_{\nu}\right)$ distribution is a convolution of the redshift and energy distributions, and hence the $\log N\left(>F_{\nu}\right)-\log F_{\nu}$ distribution is insensitive to these two distributions [6, 9, 38 40]. So, the following strategy was used in [38]: For each redshift distribution, one can adjust the $E$ distribution model $\left(\alpha\right.$ and $\left.E_{c}\right)$ and the sensitivity model $\left(\log F_{\nu \text {, th }}^{\max }\right)$ to make the $\log F_{\nu}$ distribution of the mock "observed" sample being not rejected by the KS test against the observational $\log F_{\nu}$ distribution. Once $\alpha, E_{c}$, and $\log F_{\nu \text {, th }}^{\max }$ are determined by the $\log F_{\nu}$ criterion, one can then evaluate the $\log E$ and $\mathrm{DM}_{\mathrm{E}}$ distribution criteria. The redshift distribution model is ruled out if the same mock FRB sample fails both $\log E$ and $\mathrm{DM}_{\mathrm{E}}$ criteria [38].

The above strategy of [38] is efficient in the case of unfixed $E$ distribution model, since adjusting the free parameters $\alpha, E_{c}$ and $\log F_{\nu \text {, th }}^{\max }$ consumes a large amount of computational power and time. But a potential risk exists in this strategy. The $\log F_{\nu}$ criterion has a superior position. If one tends to find a fairly high $p_{\mathrm{KS}}$ (or a fairly low $D_{\mathrm{KS}}$ ) for the $\log F_{\nu}$ criterion (say, $p_{\mathrm{KS}}>0.9$ or 0.8 ), the cases with three $p_{\mathrm{KS}}$ being all larger than (say) 0.5 or 0.4 for the $\log F_{\nu}, \log E, \mathrm{DM}_{\mathrm{E}}$ criteria might be ignored. Many acceptable cases with three not so high but still enough $p_{\mathrm{KS}}$ might not be found in this strategy.

In the present work, we use a different strategy. As mentioned at the beginning of Sec. IIA, we fix the $E$ distribution model with $\alpha=1.9$ and $\log E_{c}=41$, in order to save the computational power and time. So, we can simultaneously scan the parameters in the redshift distribution model and the sensitivity model $\left(\log F_{\nu, \mathrm{th}}^{\max }\right)$ to evaluate all the three $p_{\mathrm{KS}}$ for the $\log F_{\nu}, \log E$ and $\mathrm{DM}_{\mathrm{E}}$ criteria. In our strategy, the $\log F_{\nu}$ criterion has no superior position. In this way, we might find some acceptable cases with three high enough $p_{\mathrm{KS}}($ say, all $>0.5)$ for the $\log F_{\nu}, \log E$ and $\mathrm{DM}_{\mathrm{E}}$ criteria at the same time.

\section{VARIOUS FRB DISTRIBUTIONS VERSUS THE FIRST CHIME/FRB CATALOG}

\section{A. Various empirical FRB redshift distribution models}

History always repeats itself. In the reviews e.g. [4, 8], it was argued that FRBs might share a similar research history with gamma-ray bursts (GRBs). In [38], it is found that CHIME/FRB population does not track SFH. This also happened in the field of GRBs many years ago 77]. The enhanced evolution in the GRB rate can be parameterized as $d N /(d t d V) \propto \mathcal{E}(z) \cdot \mathrm{SFH}(z)$ [7] (see also e.g. 65]) with $\mathcal{E}(z) \propto(1+z)^{1.5}$. This motivates us to also consider some SFH-based redshift distribution models for FRBs, namely

$$
d N /(d t d V) \propto \mathcal{E}(z) \cdot \operatorname{SFH}(z)
$$

where $\operatorname{SFH}(z)$ is given by Eq. (5). Note that these models in Eq. (10) with various $\mathcal{E}(z)$ considered in the present work are all phenomenological models. $\mathcal{E}(z)$ characterizes the deviation from SFH. They are motivated by the case of GRBs, but just in the functional form, rather than the physical meaning. Although $\mathcal{E}(z) \propto(1+z)^{1.5}$ in the case of GRBs indicates an enhanced evolution, we stress that $\mathcal{E}(z)$ in the case of FRBs can be fairly different in fact, which will be determined only by the observational data. Actually, a suppressed evolution cannot be excluded in the case of FRBs. In this work, we consider the following models in detail: 


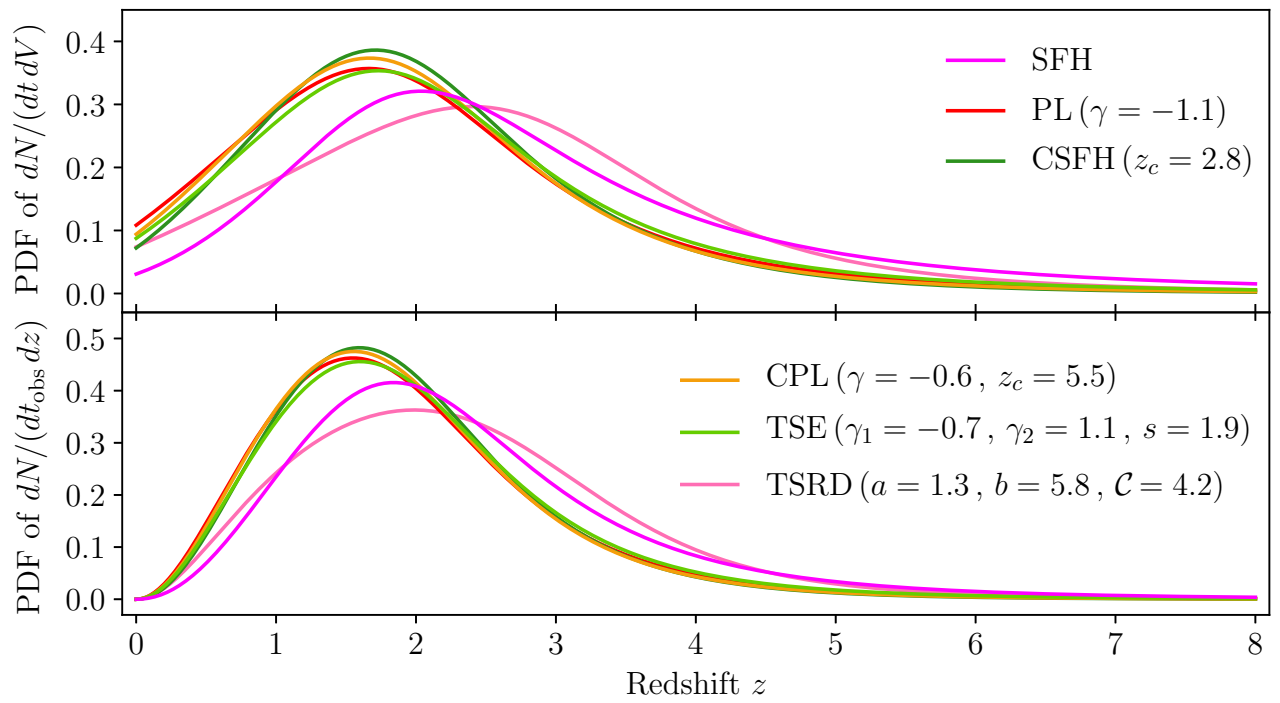

FIG. 1: Normalized probability distribution functions (PDFs) of the intrinsic FRB event rate density $d N /(d t d V)$ (upper panel) and the observed FRB event rate redshift distribution $d N /\left(d t_{\text {obs }} d V\right)$ (lower panel) for various models (color online) with some demonstrative model parameters. Notice that they are all model curves predicted by Eqs. (10) or (14) for $d N /(d t d V)$ and Eq. (2) for $d N /\left(d t_{\text {obs }} d V\right)$. No sensitivity threshold and instrumental selection effects have been applied in plotting Fig. 1 See Sec. III for details.

- $\mathrm{SFH}$ model, $\mathcal{E}(z) \equiv 1$;

- Power law $\mathcal{E}(z)(\mathrm{PL})$ model, namely

$$
\mathcal{E}(z) \propto(1+z)^{\gamma}
$$

which reduces to the SFH model if $\gamma=0$;

- Power law $\mathcal{E}(z)$ with an exponential cutoff (CPL) model, namely

$$
\mathcal{E}(z) \propto(1+z)^{\gamma} \exp \left(-z / z_{c}\right)
$$

- SFH with an exponential cutoff $(\mathrm{CSFH})$ model, i.e. the special case of CPL model with $\gamma=0$;

- Two-segment $\mathcal{E}(z)(\mathrm{TSE})$ model, namely

$$
\mathcal{E}(z) \propto \frac{(1+z)^{\gamma_{1}}}{1+((1+z) / s)^{\gamma_{1}+\gamma_{2}}}
$$

which is expected to behave like $(1+z)^{\gamma_{1}}$ and $(1+z)^{-\gamma_{2}}$ at low- and high-redshifts respectively, if $s$ is much larger than 1 and $\gamma_{1}+\gamma_{2}$ is positive.

In fact, $\operatorname{SFH}(z)$ given by Eq. (5) also behaves like $(1+z)^{2.6}$ and $(1+z)^{-3.6}$ at low- and high-redshifts, respectively. In the PL and CPL models, the two segments of SFH are changed in the same way. In the TSE model, they are changed in different ways. This inspires us to directly consider a non-SFH-based model at the same level of Eq. (10), namely

- Two-segment redshift distribution (TSRD) model,

$$
d N /(d t d V) \propto \frac{(1+z)^{a}}{1+((1+z) / \mathcal{C})^{a+b}},
$$

which cannot be confused with the TSE model. It is expected to behave like $(1+z)^{a}$ and $(1+z)^{-b}$ at low- and high-redshifts respectively, if $\mathcal{C}$ is much larger than 1 and $a+b$ is positive. 



FIG. 2: Testing the SFH model against the CHIME/FRB data (color online), with respect to the (a) $\log F_{\nu}$, (b) $\log E$ and (c) $\mathrm{DM}_{\mathrm{E}}$ criteria. In panels (a), (b) and (c), the simulations are scaled to the CHIME/FRB data. The p-values $p_{\mathrm{KS}}$ for the KS tests are given in the corresponding panels, respectively. In panel (d), the DM $\mathrm{E}_{\mathrm{E}}-\log E$ distribution is also presented for illustration (not for test). See the text for details.

In Fig. 1, we show the normalized probability distribution functions (PDFs) of the intrinsic FRB event rate density $d N /(d t d V)$ and the observed FRB event rate redshift distribution $d N /\left(d t_{\mathrm{obs}} d V\right)$ for various models with some demonstrative model parameters. Notice that they are all model curves predicted by Eqs. (10) or (14) for $d N /(d t d V)$ and Eq. (2) for $d N /\left(d t_{\text {obs }} d V\right)$. No sensitivity threshold and instrumental selection effects have been applied in plotting Fig. 1. Clearly, all models have two-segment shapes, and peak around redshift $1.5 \sim 2.5$. However, if we further consider the sensitivity threshold and instrumental selection effects mentioned at the end of Sec. IIA these distributions will be changed accordingly.

\section{B. Results}

Here, we test the six FRB redshift distribution models proposed in Sec. IIIA against the CHIME/FRB observational data. We perform the Monte Carlo simulations following the description given in Sec. IIA, and filter the mock FRBs with the sensitivity threshold and instrumental selection effects to obtain a mock "observed" sample of FRBs as mentioned at the end of Sec. IIA In our strategy, we simultaneously scan the free parameters in the redshift distribution model, within some suitable value ranges for these parameters, as mentioned in Sec. IIC For each value combination of the free parameters in the redshift distribution model, we generate 2,000,000 (or more if necessary) mock FRBs to ensure that there are still enough FRBs filtered by the sensitivity threshold and instrumental selection effects (say, $N>300$ ), comparable with the CHIME/FRB sample consisting of 452 FRBs (and we strongly refer to Sec. IV] for the discussion about the computation cost). The KS tests are performed between the mock "observed" sample of FRBs and the CHIME/FRB observational sample, with respect to the $\log F_{\nu}, \log E$ and $\mathrm{DM}_{\mathrm{E}}$ distributions. In the present work, for the CHIME/FRB data, we choose 20 bins in $\log F_{\nu}$ covering a 


\begin{tabular}{ccccc}
\hline \hline$\gamma$ & $\log F_{\nu, \mathrm{th}}^{\max }$ & $p_{\mathrm{KS}}$ for $\log F_{\nu}$ & $p_{\mathrm{KS}}$ for $\log E$ & $p_{\mathrm{KS}}$ for $\mathrm{DM}_{\mathrm{E}}$ \\
\hline $\mathbf{- 1 . 1}$ & $\mathbf{0 . 7 2}$ & $\mathbf{0 . 9 4 7 8}$ & $\mathbf{0 . 4 9 0 5}$ & $\mathbf{0 . 5 5 4 8}$ \\
\hline-0.9 & 0.75 & 0.8697 & 0.2179 & 0.3280 \\
\hline-0.8 & 0.76 & 0.8468 & 0.2257 & 0.5889 \\
\hline-0.7 & 0.68 & 0.8733 & 0.2333 & 0.3671 \\
\hline \hline
\end{tabular}

TABLE I: Some examples of the acceptable PL models with the redshift distribution model parameter $\gamma$ and the sensitivity model parameter $\log F_{\nu, \mathrm{th}}^{\max }$, as well as three $p_{\mathrm{KS}}$ for the $\log F_{\nu}, \log E$ and $\mathrm{DM}_{\mathrm{E}}$ criteria against the CHIME/FRB data. The boldfaced ones are also presented in the accompanying plots. See Sec. IIIB for details.
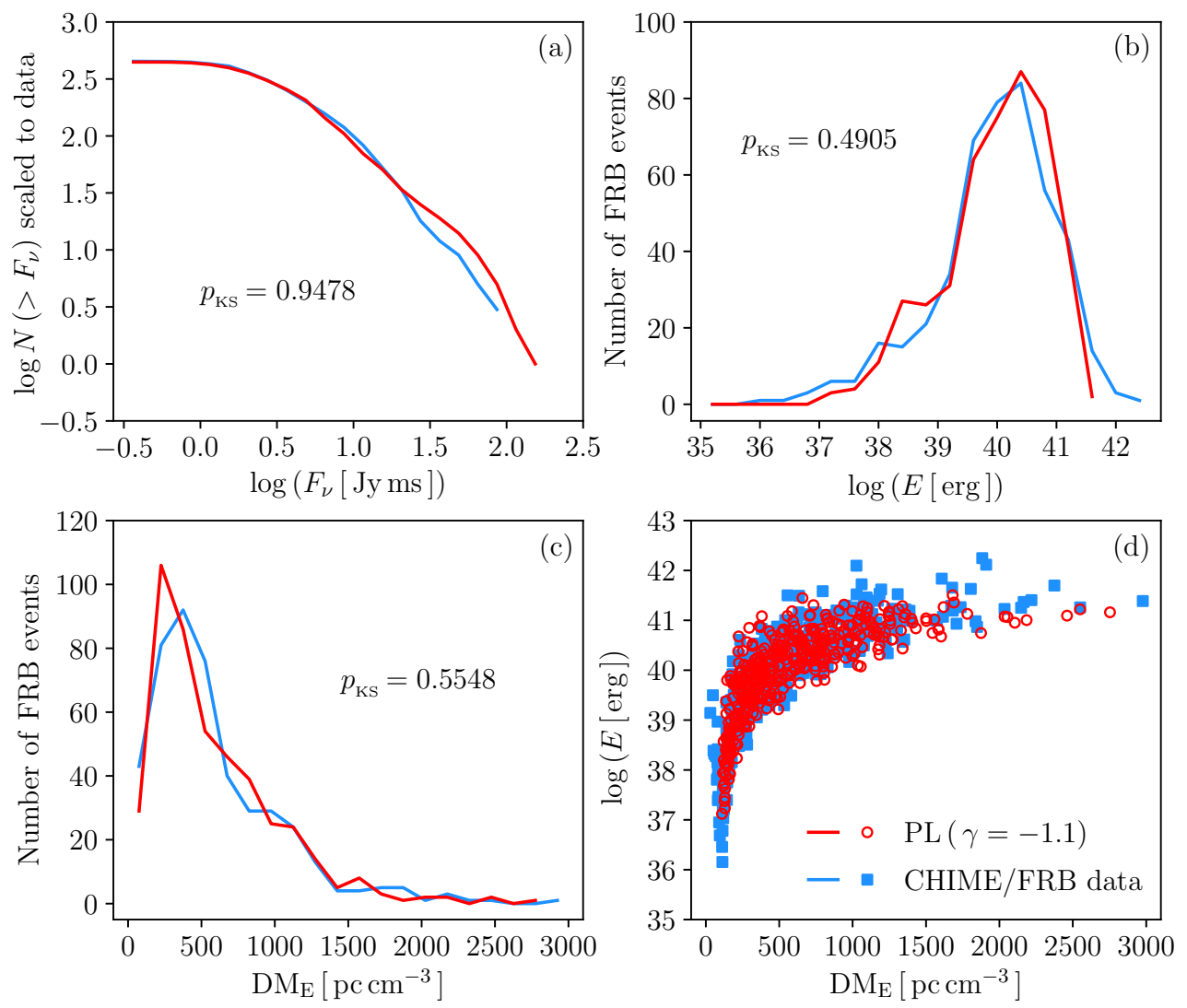

FIG. 3: The same as in Fig. 2 but for the PL model with the model parameter given in panel (d).

range from -0.5 to 2.0 , in $\log E$ covering a range from 35 to 43 , in $\mathrm{DM}_{\mathrm{E}}$ covering a range from 0 to $3000 \mathrm{pc} \mathrm{cm}^{-3}$, respectively. For the simulations, if necessary, we add bins with the same bin size.

At first, we consider the SFH model in which the FRB distribution tracks SFH (namely $\mathcal{E}(z) \equiv 1$ in Eq. (10) while $\operatorname{SFH}(z)$ is given by Eq. (50). In this case, there is no free parameter in the FRB redshift distribution model. Noting that 2,000,000 mock FRBs are not enough to leave more than 300 FRBs filtered by the sensitivity threshold and instrumental selection effects, we have to generate $5,000,000$ mock FRBs in the simulation. We scan the free parameter $\log F_{\nu \text {, th }}^{\max }$ in the sensitivity model from -0.49 to 2.0 with step size 0.01 , and evaluate three p-values of the KS tests with respect to the $\log F_{\nu}, \log E$ and $\mathrm{DM}_{\mathrm{E}}$ criteria for each value of $\log F_{\nu, \mathrm{th}}^{\max }$. We find that the $\mathrm{SFH}$ model fails to reproduce all the $\log F_{\nu}$, $\log E$ and $\mathrm{DM}_{\mathrm{E}}$ distributions of the CHIME/FRB sample, because for all values of $\log F_{\nu, \mathrm{th}}^{\max }$, three $p_{\mathrm{KS}}$ cannot be simultaneously larger than 0.1. In Fig. 22, we present a typical example with $\log F_{\nu, \text { th }}^{\max }=0.81$. Its KS p-values for both $\log E$ and $\mathrm{DM}_{\mathrm{E}}$ criteria are $\mathcal{O}\left(10^{-4}\right) \ll 0.05$. From panel (d) of Fig. 2, one 


\begin{tabular}{cccccc}
\hline \hline$\gamma$ & $z_{c}$ & $\log F_{\nu, \mathrm{th}}^{\max }$ & $p_{\mathrm{KS}}$ for $\log F_{\nu}$ & $p_{\mathrm{KS}}$ for $\log E$ & $p_{\mathrm{KS}}$ for $\mathrm{DM}_{\mathrm{E}}$ \\
\hline $\mathbf{0 . 6}$ & $\mathbf{5 . 5}$ & $\mathbf{0 . 7 4}$ & $\mathbf{0 . 8 2 6 6}$ & $\mathbf{0 . 6 0 0 2}$ & $\mathbf{0 . 5 2 3 4}$ \\
\hline $\mathbf{0 . 2}$ & $\mathbf{2 . 7}$ & $\mathbf{0 . 7 0}$ & $\mathbf{0 . 5 4 1 9}$ & $\mathbf{0 . 6 3 5 0}$ & $\mathbf{0 . 5 0 1 5}$ \\
\hline-0.1 & 2.3 & 0.76 & 0.6893 & 0.4480 & 0.5806 \\
\hline-0.3 & 3.2 & 0.81 & 0.6375 & 0.5079 & 0.4744 \\
\hline-0.4 & 3.4 & 0.80 & 0.6469 & 0.4042 & 0.5302 \\
\hline-0.6 & 5.2 & 0.79 & 0.5802 & 0.5028 & 0.5181 \\
\hline-0.5 & 6.5 & 0.79 & 0.7272 & 0.5815 & 0.5082 \\
\hline-0.4 & 7.3 & 0.77 & 0.6147 & 0.5132 & 0.5982 \\
\hline-0.9 & 8.0 & 0.70 & 0.9063 & 0.6155 & 0.5672 \\
\hline 0.1 & 1.6 & 0.74 & 0.6627 & 0.4106 & 0.4457 \\
\hline \hline
\end{tabular}

TABLE II: The same as in Table [ but for some examples of the acceptable CPL model.
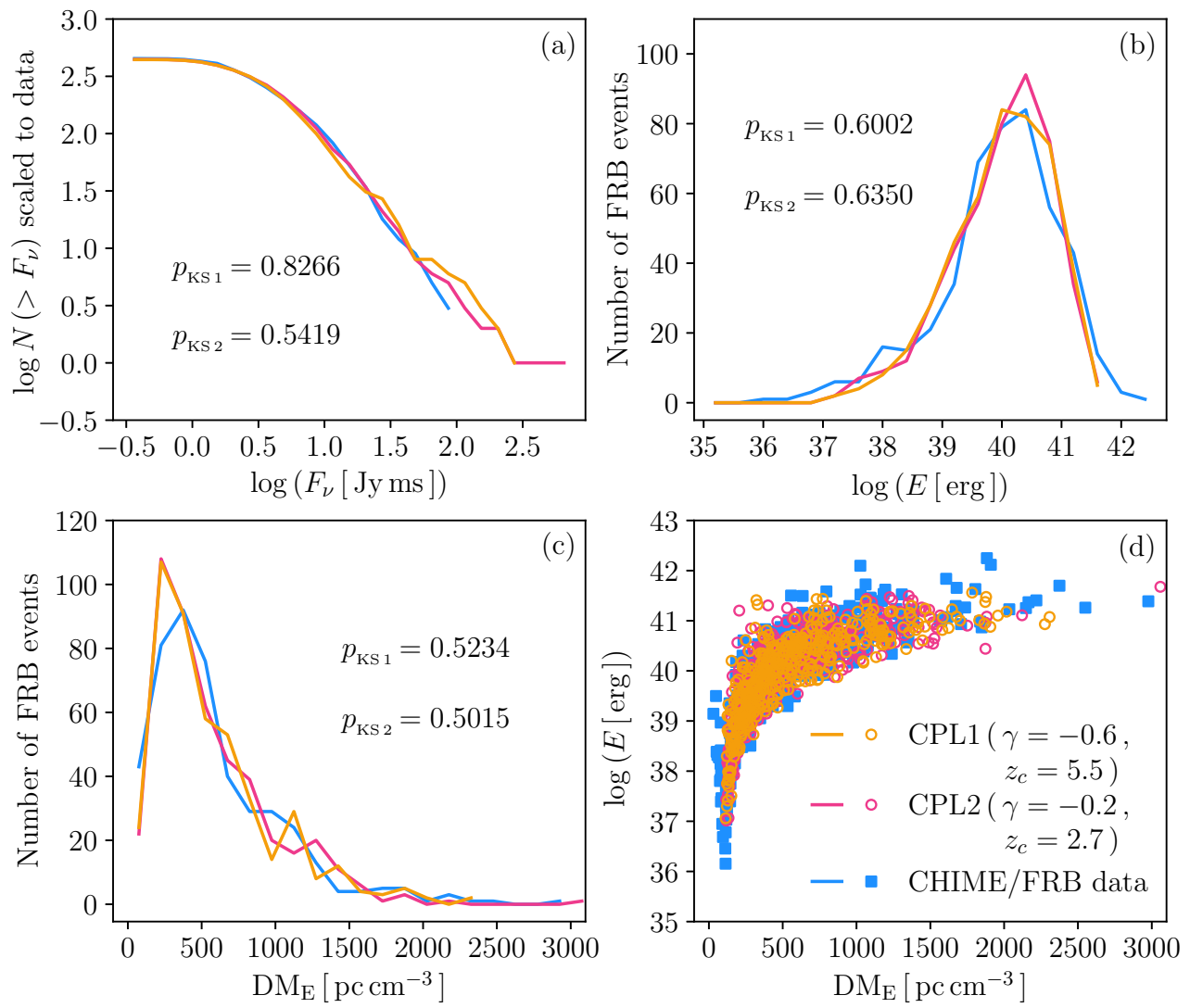

FIG. 4: The same as in Fig. 2] but for the CPL model with the model parameters given in panel (d).

can see that many mock FRBs with $\mathrm{DM}_{\mathrm{E}} \gtrsim 1000 \mathrm{pc} \mathrm{cm}^{-3}$ are clearly outside the crowded region of the CHIME/FRB observational data. From panel (c) of Fig. 2 we find that the number of mock FRBs with relatively large $\mathrm{DM}_{\mathrm{E}} \gtrsim 700 \mathrm{pc} \mathrm{cm}^{-3}$ is overproduced. This hints that a suppressed evolution at large $\mathrm{DM}_{\mathrm{E}}$ (or equivalently high-redshifts) might be required by the CHIME/FRB observational data. Clearly, due to the very low p-values $p_{\mathrm{KS}} \ll 0.05$, the CHIME/FRB observational data strongly reject the SFH 


\begin{tabular}{ccccc}
\hline \hline$z_{c}$ & $\log F_{\nu, \mathrm{th}}^{\max }$ & $p_{\mathrm{KS}}$ for $\log F_{\nu}$ & $p_{\mathrm{KS}}$ for $\log E$ & $p_{\mathrm{KS}}$ for $\mathrm{DM}_{\mathrm{E}}$ \\
\hline $\mathbf{2 . 8}$ & $\mathbf{0 . 7 0}$ & $\mathbf{0 . 9 6 1 4}$ & $\mathbf{0 . 2 6 6 2}$ & $\mathbf{0 . 5 4 7 9}$ \\
\hline 2.9 & 0.77 & 0.9003 & 0.2869 & 0.4900 \\
\hline 3.6 & 0.73 & 0.5305 & 0.2609 & 0.4994 \\
\hline 2.4 & 0.72 & 0.8368 & 0.2101 & 0.3914 \\
\hline 2.0 & 0.69 & 0.4177 & 0.2270 & 0.2526 \\
\hline \hline
\end{tabular}

TABLE III: The same as in Table I, but for some examples of the acceptable CSFH model.


FIG. 5: The same as in Fig. 2] but for the CSFH model with the model parameter given in panel (d).

model at very high confidence. We confirm the result for the SFH model found in [38]. So, one should find some empirical FRB distribution models consistent with the CHIME/FRB observational data.

As mentioned above, motivated by the similar case of GRBs, we consider some SFH-based FRB redshift distribution models given by Eq. (10). Notice that for all the following models, it is enough to generate 2,000,000 mock FRBs for each value combination of the free parameters in the redshift distribution model to leave more than $300 \mathrm{FRBs}$ filtered by the sensitivity threshold and instrumental selection effects, comparable with the CHIME/FRB sample of 452 FRBs as mentioned in Sec. IIB.

The simplest one is the PL model given by Eq. (11), which was also considered in the case of GRBs 77] (see also e.g. 65]). There is only one free parameter $\gamma$ in the FRB redshift distribution model. We scan the free parameter $\gamma$ from -2.5 to +2.5 with step size 0.1 , and scan $\log F_{\nu, \text { th }}^{\max }$ in the sensitivity model from -0.49 to 2.0 with step size 0.01 . For each parameter combination $\left(\gamma, \log F_{\nu, \text { th }}^{\max }\right)$, we evaluate three p-values of the KS tests with respect to the $\log F_{\nu}, \log E$ and $\mathrm{DM}_{\mathrm{E}}$ criteria. Some examples are shown 


\begin{tabular}{ccccccc}
\hline \hline$\gamma_{1}$ & $\gamma_{2}$ & $s$ & $\log F_{\nu, \mathrm{th}}^{\max }$ & $p_{\mathrm{KS}}$ for $\log F_{\nu}$ & $p_{\mathrm{KS}}$ for $\log E$ & $p_{\mathrm{KS}}$ for $\mathrm{DM}_{\mathrm{E}}$ \\
\hline$-\mathbf{0 . 7}$ & $\mathbf{1 . 1}$ & $\mathbf{1 . 9}$ & $\mathbf{0 . 7 6}$ & $\mathbf{0 . 9 8 2 5}$ & $\mathbf{0 . 7 4 1 4}$ & $\mathbf{0 . 7 6 0 9}$ \\
\hline $\mathbf{- 0 . 9}$ & $\mathbf{0 . 6}$ & $\mathbf{0 . 4}$ & $\mathbf{0 . 7 7}$ & $\mathbf{0 . 8 5 1 6}$ & $\mathbf{0 . 7 0 4 4}$ & $\mathbf{0 . 7 0 4 4}$ \\
\hline-1.3 & 0.6 & 0.4 & 0.75 & 0.8445 & 0.7149 & 0.7207 \\
\hline-0.3 & 1.4 & 0.7 & 0.88 & 0.7306 & 0.8934 & 0.7234 \\
\hline-1.1 & 0.4 & 0.7 & 0.77 & 0.7112 & 0.7576 & 0.7281 \\
\hline-0.9 & 0.6 & 2.4 & 0.72 & 0.9988 & 0.6401 & 0.6352 \\
\hline-1.1 & 0.8 & 3.2 & 0.76 & 0.9747 & 0.6659 & 0.6153 \\
\hline-0.2 & 2.2 & 3.6 & 0.76 & 0.9523 & 0.6461 & 0.6498 \\
\hline \hline
\end{tabular}

TABLE IV: The same as in Table \ but for some examples of the acceptable TSE model.

in Table I. We find that the PL model with some negative $\gamma$ cannot be rejected at high confidence by the CHIME/FRB observational data, with respect to all the three criteria for $\log F_{\nu}, \log E$ and $\mathrm{DM}_{\mathrm{E}}$. In particular, we present an explicit example of the PL model with $\gamma=-1.1$ (and $\log F_{\nu, \text { th }}^{\max }=0.72$ ) in Fig. 3 , whose three p-values are all larger than 0.49 simultaneously. So, the PL model with suitable parameters can be consistent with the CHIME/FRB observational data. Clearly, a negative $\gamma$ is commonly required. It is worth noting that $\mathcal{E}(z) \propto(1+z)^{\gamma}$ with a negative $\gamma$ for FRBs indicates a suppressed evolution with respect to SFH (n.b. Eq. (10) with Eq. (11)). On the contrary, as mentioned above, an enhanced evolution with respect to SFH (namely $\mathcal{E}(z) \propto(1+z)^{1.5}$ ) was found for GRBs [77] (see also e.g. [65]). These two trends are opposite in fact. This suggests that FRBs might be not closely related to GRBs, and might shed light on the origin of FRBs.

Of course, an exponential cutoff might be taken into account as in the literature. So, it is reasonable to consider the CPL model given by Eq. (12), which has two free parameters $\gamma$ and $z_{c}$. It is expected that $\mathcal{E}(z)$ has a sharp cutoff around $z_{c}$. We scan the free parameters $\gamma$ from -2.5 to +2.5 with step size $0.1, z_{c}$ from 0.1 to 8.0 with step size 0.1 , and scan $\log F_{\nu \text {, th }}^{\max }$ in the sensitivity model from -0.49 to 2.0 with step size 0.01 . For each parameter combination $\left(\gamma, z_{c}, \log F_{\nu, \text { th }}^{\max }\right)$, we evaluate three p-values of the KS tests with respect to the $\log F_{\nu}, \log E$ and $\mathrm{DM}_{\mathrm{E}}$ criteria. Some examples are shown in Table II] We find that the CPL model with suitable parameters cannot be rejected by the CHIME/FRB observational data, with respect to all the three criteria for $\log F_{\nu}, \log E$ and $\mathrm{DM}_{\mathrm{E}}$. For a very high $z_{c}$ (say, $>7$ ), the CPL model effectively reduces to the PL model, since there are only a few FRBs at very high redshifts. As shown in Table III some moderate $z_{c}$ are still viable, and in these cases their corresponding $\gamma$ need not to be very negative. In Fig. 4, we present two explicit examples of the CPL model with different model parameters. Obviously, the CPL model with suitable parameters can also be consistent with the CHIME/FRB observational data. For many parameters, three p-values for the $\log F_{\nu}, \log E$ and $\mathrm{DM}_{\mathrm{E}}$ criteria are all larger than 0.5 simultaneously. Comparing three $p_{\mathrm{KS}}$ in Table $\amalg$ with the ones in Table【. the CPL model is notably favored over the PL model, since all the three p-values for the $\log F_{\nu}, \log E$ and $\mathrm{DM}_{\mathrm{E}}$ criteria are closer to 1 , especially for both $\log E$ and $\mathrm{DM}_{\mathrm{E}}$ criteria. Note that a negative $\gamma$ in the CPL model is commonly required by the CHIME/FRB observational data, which indicates a suppressed evolution with respect to $\mathrm{SFH}$ again.

A special case of the CPL model is of interest. If $\gamma=0$ in the CPL model, it becomes SFH with an exponential cutoff (CSFH) model. It might loosely track SFH at low-redshifts if the sharp cutoff appears around a fairly high $z_{c}$. We scan the only free parameter $z_{c}$ in $\mathcal{E}(z)$ from 0.1 to 8.0 with step size 0.1 , and scan $\log F_{\nu \text {, th }}^{\max }$ in the sensitivity model from -0.49 to 2.0 with step size 0.01 . For each parameter combination $\left(z_{c}, \log F_{\nu, \mathrm{th}}^{\max }\right)$, we evaluate three p-values of the KS tests with respect to the $\log F_{\nu}, \log E$ and $\mathrm{DM}_{\mathrm{E}}$ criteria. As shown in Table III and Fig. [5. the CSFH model with suitable parameters cannot be rejected at high confidence by the $\mathrm{CHIME} / \mathrm{FRB}$ observational data, with respect to all the three criteria for $\log F_{\nu}, \log E$ and $\mathrm{DM}_{\mathrm{E}}$. This might be welcome to some theoretical models for the FRB engines, in which FRBs still track SFH at low-redshifts, only at the price of breaking down at fairly high-redshifts, where some unknown factors necessary to trigger FRBs might be not ready. 

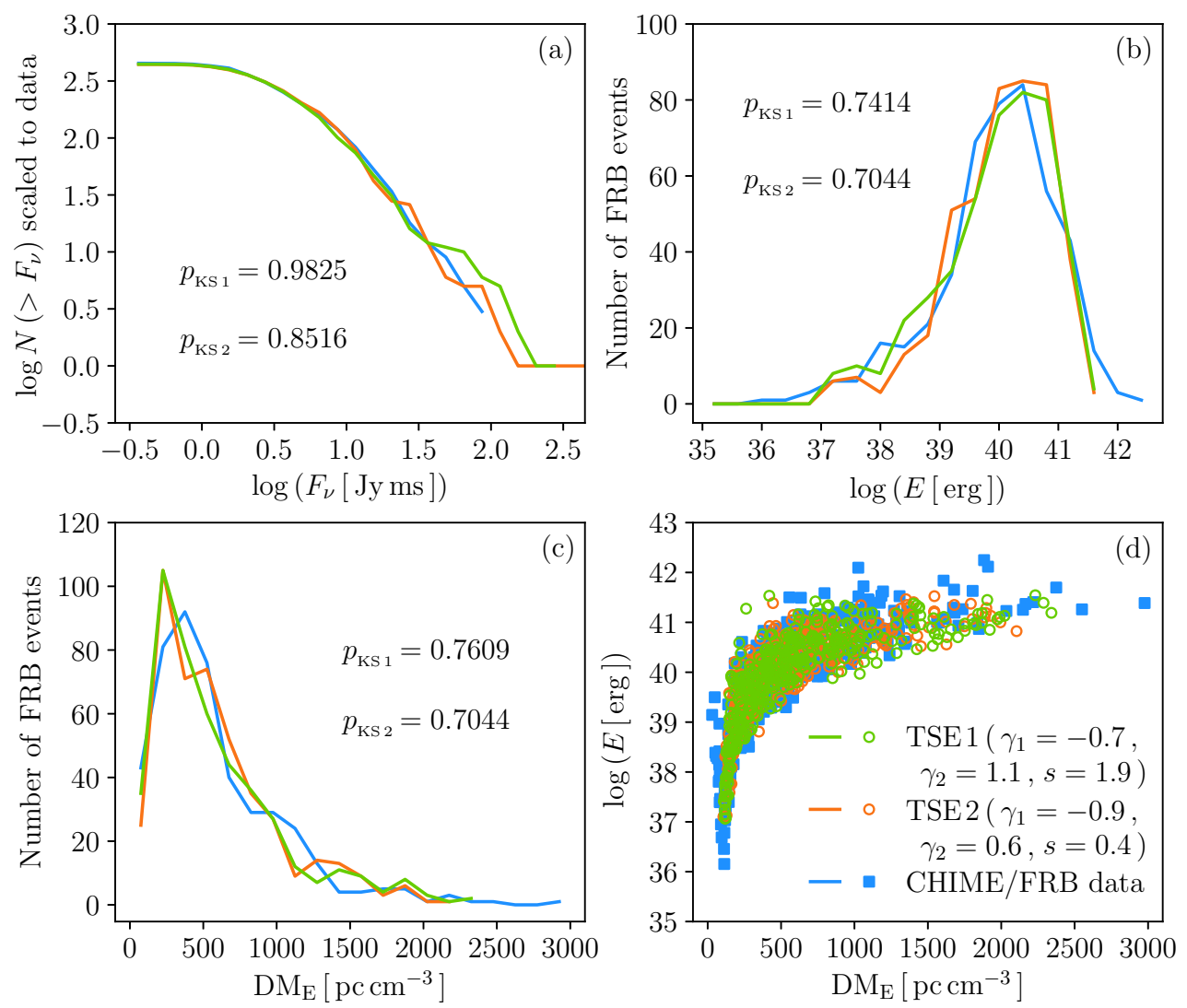

FIG. 6: The same as in Fig. 2] but for the TSE model with the model parameters given in panel (d).

In the TSE model given by Eq. (13), due to the two-segment behavior of $\mathcal{E}(z)$, the evolution with respect to SFH might be enhanced (suppressed) at low-redshifts, and suppressed (enhanced) at high-redshifts, respectively. Of course, it is also possible to be suppressed (enhanced) at both low- and high-redshifts but with different power law indices. There are three free parameters $\gamma_{1}, \gamma_{2}$ and $s$ in $\mathcal{E}(z)$. We scan $\gamma_{1}$ from -2.0 to $+2.0, \gamma_{2}$ from -3.0 to $+3.0, s$ from 0.1 to 4.0 . To save the computational power and time, we firstly scan them with a rough step size 0.2 , and then re-scan them with a finer step size 0.1 in the parameter spaces having high p-values. For each parameter combination $\left(\gamma_{1}, \gamma_{2}, s\right)$, we scan $\log F_{\nu, \text { th }}^{\max }$ in the sensitivity model from -0.49 to 2.0 with step size 0.01 and evaluate three p-values of the KS tests with respect to the $\log F_{\nu}, \log E$ and $\mathrm{DM}_{\mathrm{E}}$ criteria. Some examples are shown in Table IV. We find that the TSE model with suitable parameters cannot be rejected by the CHIME/FRB observational data, with respect to all the three criteria for $\log F_{\nu}, \log E$ and $\mathrm{DM}_{\mathrm{E}}$. As shown in Table IV for many parameters, three p-values for the $\log F_{\nu}, \log E$ and $\mathrm{DM}_{\mathrm{E}}$ criteria are all larger than 0.7 simultaneously. In fact, there are more examples with three p-values all larger than 0.6 simultaneously, and we have not clearly shown them in Table IV. In Fig. 6. we present two explicit examples of the TSE model with different model parameters. Obviously, the TSE model with suitable parameters can be fully consistent with the CHIME/FRB observational data. Comparing three $p_{\mathrm{KS}}$ in Table IV with the ones in Table II the TSE model is notably favored over the CPL model, since all the three p-values for the $\log F_{\nu}, \log E$ and $\mathrm{DM}_{\mathrm{E}}$ criteria are closer to 1 . Note that a negative $\gamma_{1}$ and a positive $\gamma_{2}$ in the TSE model are simultaneously favored by the CHIME/FRB observational data, which indicate a suppressed evolution with respect to $\mathrm{SFH}$ at both low- and high-redshifts.

Since $\operatorname{SFH}(z)$ in Eq. (5) has also a two-segment behavior, and $\mathcal{E}(z)$ in Eq. (10) suppresses (enhances) it with different power law indices at low- and high-redshifts, we can rather parameterize $d N /(d t d V)$ directly by using a single two-segment function given by Eq. (14). There are three free parameters $a, b$ and $\mathcal{C}$ in this non-SFH-based TSRD model. We scan $a$ from -2.0 to $+3.0, b$ from -3.0 to $+6.0, \mathcal{C}$ from 0.1 to 5.0. To save the computational power and time, similar to the TSE model, we firstly scan them with a rough step size 0.2 , and then re-scan them with a finer step size 0.1 in the parameter spaces having high 


\begin{tabular}{ccccccc}
\hline \hline$a$ & $b$ & $\mathcal{C}$ & $\log F_{\nu, \mathrm{th}}^{\max }$ & $p_{\mathrm{KS}}$ for $\log F_{\nu}$ & $p_{\mathrm{KS}}$ for $\log E$ & $p_{\mathrm{KS}}$ for $\mathrm{DM}_{\mathrm{E}}$ \\
\hline $\mathbf{1 . 3}$ & $\mathbf{5 . 8}$ & $\mathbf{4 . 2}$ & $\mathbf{0 . 7 1}$ & $\mathbf{0 . 9 5 0 2}$ & $\mathbf{0 . 9 2 1 0}$ & $\mathbf{0 . 8 0 2 0}$ \\
\hline $\mathbf{1 . 4}$ & $\mathbf{2 . 4}$ & $\mathbf{3 . 7}$ & $\mathbf{0 . 6 6}$ & $\mathbf{0 . 9 6 1 6}$ & $\mathbf{0 . 7 7 3 4}$ & $\mathbf{0 . 8 1 6 1}$ \\
\hline 1.1 & 2.7 & 4.3 & 0.72 & 0.7670 & 0.8397 & 0.7874 \\
\hline 0.9 & 3.5 & 4.8 & 0.72 & 0.7748 & 0.7801 & 0.8532 \\
\hline 1.4 & 2.8 & 4.1 & 0.61 & 0.9215 & 0.7611 & 0.7717 \\
\hline 1.3 & 2.3 & 5.0 & 0.56 & 0.8841 & 0.8288 & 0.7174 \\
\hline 1.6 & 1.3 & 2.8 & 0.69 & 0.8816 & 0.7396 & 0.7722 \\
\hline 1.2 & 4.7 & 4.1 & 0.72 & 0.8210 & 0.7945 & 0.7174 \\
\hline \hline
\end{tabular}

TABLE V: The same as in Table [ but for some examples of the acceptable TSRD model.

p-values. For each parameter combination $(a, b, \mathcal{C})$, we scan $\log F_{\nu, \text { th }}^{\max }$ in the sensitivity model from -0.49 to 2.0 with step size 0.01 and evaluate three p-values of the KS tests with respect to the $\log F_{\nu}, \log E$ and $\mathrm{DM}_{\mathrm{E}}$ criteria. Some examples are shown in Table $\mathrm{V}$. For many parameters, three p-values for the $\log F_{\nu}, \log E$ and $\mathrm{DM}_{\mathrm{E}}$ criteria are all larger than 0.75 simultaneously (all $>0.8$ for a few parameters). In Fig. 7, we present two explicit examples of the TSRD model with different model parameters. Clearly, the TSRD model with suitable parameters can be fully consistent with the CHIME/FRB observational data. Comparing three $p_{\mathrm{KS}}$ in Table $\mathrm{V}$ with the ones in Table IV the TSRD model is slightly favored over the TSE model, since all the three p-values for the $\log F_{\nu}, \log E$ and $\mathrm{DM}_{\mathrm{E}}$ criteria are closer to 1 . For the most favored TSRD parameters $a=1.3, b=5.8$ and $\mathcal{C}=4.2$ in Table $\nabla$, $d N /(d t d V)$ in Eq. (14) behaves like $(1+z)^{1.3}$ and $(1+z)^{-5.8}$ at low- and high-redshifts, respectively. Noting that $\operatorname{SFH}(z)$ in Eq. (5) behaves like $(1+z)^{2.6}$ and $(1+z)^{-3.6}$ at low- and high-redshifts respectively, the most favored TSRD model in Table $\mathrm{V}$ is suppressed with respect to SFH at both low- and high-redshifts. However, since the TSRD model is not based on SFH, it is hard to directly compare the TSRD model with the $\mathrm{SFH}$ model in general. Although all $p_{\mathrm{KS}}>0.75$ examples in Table $\mathrm{V}$ have $b>a>0$, there are still many acceptable cases with quite different parameters, e.g. both negative $a$ and $b$ (which are not presented in Table $\mathrm{V}$ ), but their three $p_{\mathrm{KS}}>0.6$ or 0.5 simultaneously. For those parameters, the TSRD model has different behaviors. In fact, the CHIME/FRB observational data can be consistent with quite different TSRD models. A much larger observational sample is required to differentiate them.

In brief, we have tested six types of FRB redshift distribution models, and found that many of them can be fully consistent with the CHIME/FRB observational data for some suitable model parameters. According to three KS p-values for the $\log F_{\nu}, \log E$ and $\mathrm{DM}_{\mathrm{E}}$ criteria, they are favored in the following order: TSRD $\gtrsim \mathrm{TSE}>\mathrm{CPL}>\mathrm{PL}>\mathrm{CSFH} \gg \mathrm{SFH}$. Noting that they have 3, 3, 2, 1, 1, 0 free parameters in the redshift distribution models respectively, this order is reasonable in fact.

\section{CONCLUDING REMARKS}

Nowadays, FRBs have become one of the active fields in astronomy and cosmology. However, the origin of FRBs is still unknown to date. The studies on the intrinsic FRB distributions might help us to reveal the possible origins of FRBs, and improve the simulations for FRB cosmology. Recently, the first CHIME/FRB catalog of 536 events was released. Such a large uniform sample of FRBs detected by a single telescope is very valuable to test the FRB distributions. Later, it has been claimed that the FRB distribution model tracking SFH was rejected by the first CHIME/FRB catalog. In the present work, we consider some empirical FRB distribution models, and find that many of them can be fully consistent with the CHIME/FRB observational data for some suitable model parameters.

Some remarks are in order. At first, as shown in this work, we independently test the FRB distribution model tracking SFH against the first CHIME/FRB observational data, and affirmatively find that it can be rejected at very high confidence. We confirm this important result found in [38]. It clearly means that other FRB distribution models are required by the observational data. 



FIG. 7: The same as in Fig. 2 but for the TSRD model with the model parameters given in panel (d).

In most of the models considered here, a suppressed evolution with respect to SFH is found for FRBs. A negative power law index $\gamma$ in the PL and CPL models is commonly required by the CHIME/FRB observational data. An exponential cutoff in the CPL and CSFH models is actually a sharp suppression. A negative $\gamma_{1}$ and a positive $\gamma_{2}$ in the TSE model are simultaneously favored by the CHIME/FRB observational data, which also indicate a suppressed evolution with respect to SFH for FRBs. The most favored TSRD model is also suppressed with respect to SFH, as mentioned above. On the contrary, an enhanced evolution with respect to SFH was found for GRBs [77] (see also e.g. 65]). This suggests that FRBs might be not closely related to GRBs, and might shed light on the origin of FRBs.

On the other hand, the CSFH model with suitable parameters cannot be rejected at high confidence by the CHIME/FRB observational data, although the corresponding KS p-values are not so high. This might be welcome to some theoretical models for the FRB engines, in which FRBs still track SFH at low-redshifts, only at the price of breaking down at fairly high-redshifts, where some unknown factors necessary to trigger FRBs might be not ready.

In fact, all the "successful" models mentioned above effectively require a certain degree of "delay" with respect to SFH. This point can be clearly seen from Fig. 1. The peaks of these distribution models are effectively shifted to the low-redshifts. In some sense, our phenomenological models are in general consistent with the conclusion of [38], namely a certain degree of "delay" with respect to SFH is required by the observational data.

The main difference between the present work and [38] is that the latter claimed a much more delayed population than ours. Note that a three-segment empirical SFH model from [65] (which also includes GRB contribution from high-redshifts) was used in [38], while we instead use the two-segment empirical SFH model from [67] (see also [66]) in the present work. This might be the main reason for the much more delay required by [38] to match the same data.

It is worth noting that our FRB distribution models are mainly empirical or phenomenological ones. The physical mechanisms behind them are not clear. In the case of GRBs, an enhanced evolution with respect to SFH was well motivated, namely stars formed at higher redshifts tend to have lower metallicity 
which would facilitate generating more long GRBs (see e.g. [77-79]). However, the trend is opposite in the case of FRBs. To date, around 20 FRB host galaxies were identified [37], whose metallicities are in the range from 8.08 to 9.03 (most of them are larger than 8.7, the metallicity upper cutoff for long GRBs [79]). It seems that higher metallicity might facilitate generating more FRBs or their (unknown) progenitors (but correlation does not mean causality). As mentioned above, all the "successful" models effectively require a certain degree of "delay" with respect to SFH. Such kind of delay might rise from various physical reasons. One of the physically motivated models might be the compact binary merger models considered in e.g. [14, 38, 61, 62]. A binary system must undergo a long inspiral phase before the final merger, and hence a significant delay is necessary [14, 38]. On the other hand, the models invoking AGNs, white dwarfs, cosmic comb, black holes, cosmic strings, or interactions between asteroids/comets and neutron stars, also require a delay less than the one of compact binary merger models [14, 38]. These FRB models that involve a delay with respect to $\mathrm{SFH}$ might be regarded as the physical motivations for our distribution models considered here.

In this work, for each value combination of the free parameters in the redshift distribution model, we generate 2,000,000 mock FRBs (or 5,000,000 mock FRBs for the SFH model) to ensure that there are still enough FRBs filtered by the sensitivity threshold and instrumental selection effects (say, $N>300$ ), comparable with the CHIME/FRB sample consisting of 452 FRBs as mentioned in Sec. IIB. For example, in the CPL model having two free model parameters, we scan the free parameters $\gamma$ from -2.5 to +2.5 with step size $0.1, z_{c}$ from 0.1 to 8.0 with step size 0.1 , and hence there are about $50 \times 80=4000$ value combinations of $\left(\gamma, z_{c}\right)$. Thus, we should generate about $4000 \times 2,000,000=8,000,000,000$ mock FRBs in total. For the TSE and TSRD models having three free model parameters, the cost of computation increases dramatically, as expected. Clearly, this consumes a large amount of computational power and time. Therefore, we have to scan the model parameters roughly (with a step size 0.1 commonly) in the relatively narrow parameter spaces (especially for the TSE and TSRD models) to save the computational power and time. In this way, we might miss the optimal parameters with maximum p-values for the KS tests. But at least we do show that the FRB distribution models considered in this work can be fully consistent with the CHIME/FRB observational data for some explicit parameters. Of course, if we could use some significantly powerful computing resources to this end, it is expected that the best model parameters will be precisely found. We hope us or someone else can do that eventually in the future.

\section{ACKNOWLEDGEMENTS}

We thank the anonymous referee for quite useful comments and suggestions, which helped us to improve this work. We are grateful to Hua-Kai Deng, Han-Yue Guo, Shupeng Song, Zhong-Xi Yu and Jing-Yi Jia for kind help and useful discussions. This work was supported in part by NSFC under Grants No. 11975046 and No. 11575022.

[1] https://www.nature.com/collections/rswtktxcln

[2] D. R. Lorimer, Nat. Astron. 2, 860 (2018) arXiv:1811.00195.

[3] E. F. Keane, Nat. Astron. 2, 865 (2018) arXiv:1811.00899.

[4] S. R. Kulkarni, Nat. Astron. 2, 832 (2018) arXiv:1811.00448.

[5] U. L. Pen, Nat. Astron. 2, 842 (2018) arXiv:1811.00605.

[6] E. Petroff, J. W. T. Hessels and D. R. Lorimer, Astron. Astrophys. Rev. 27, 4 (2019) arXiv:1904.07947.

[7] J. M. Cordes and S. Chatterjee, Ann. Rev. Astron. Astrophys. 57, 417 (2019) arXiv:1906.05878.

[8] B. Zhang, Nature 587, 45 (2020) arXiv:2011.03500.

[9] D. Xiao, F. Y. Wang and Z. G. Dai, Sci. China Phys. Mech. Astron. 64, 249501 (2021) arXiv:2101.04907.

[10] E. Petroff, J. W. T. Hessels and D. R. Lorimer, arXiv:2107.10113 [astro-ph.HE].

[11] D. R. Lorimer et al., Science 318, 777 (2007) arXiv:0709.4301.

[12] D. Thornton et al., Science 341, 53 (2013) arXiv:1307.1628.

[13] E. Platts et al., Phys. Rept. 821, 1 (2019) arXiv:1810.05836; The up-to-date FRB theory catalogue is available at https://frbtheorycat.org 
[14] R. C. Zhang et al., Mon. Not. Roy. Astron. Soc. 501, 157 (2021) arXiv:2011.06151].

[15] W. Deng and B. Zhang, Astrophys. J. 783, L35 (2014) arXiv:1401.0059.

[16] Y. P. Yang and B. Zhang, Astrophys. J. 830, no. 2, L31 (2016) arXiv:1608.08154].

[17] H. Gao, Z. Li and B. Zhang, Astrophys. J. 788, 189 (2014) arXiv:1402.2498.

[18] B. Zhou, X. Li, T. Wang, Y. Z. Fan and D. M. Wei, Phys. Rev. D 89, 107303 (2014) arXiv:1401.2927.

[19] Y. P. Yang, R. Luo, Z. Li and B. Zhang, Astrophys. J. 839, no. 2, L25 (2017) arXiv:1701.06465.

[20] H. Yu and F. Y. Wang, Astron. Astrophys. 606, A3 (2017) arXiv:1708.06905.

[21] J. J. Wei, X. F. Wu and H. Gao, Astrophys. J. 860, no. 1, L7 (2018) arXiv:1805.12265.

[22] Z. X. Li, H. Gao, X. H. Ding, G. J. Wang and B. Zhang, Nat. Comm. 9, 3833 (2018) arXiv:1708.06357.

[23] M. Jaroszynski, Mon. Not. Roy. Astron. Soc. 484, no. 2, 1637 (2019) arXiv:1812.11936.

[24] M. S. Madhavacheril et al., Phys. Rev. D 100, no. 10, 103532 (2019) arXiv:1901.02418.

[25] A. Walters et al., Astrophys. J. 856, no. 1, 65 (2018) arXiv:1711.11277.

[26] Z. X. Li et al., Astrophys. J. 876, no. 2, 146 (2019) arXiv:1904.08927.

[27] J. J. Wei et al., JCAP 1909, 039 (2019) arXiv:1907.09772.

[28] R. G. Cai, T. B. Liu, S. J. Wang and W. T. Xu, JCAP 1909, 016 (2019) arXiv:1905.01803.

[29] Y. K. Wang and F. Y. Wang, Astron. Astrophys. 614, A50 (2018) arXiv:1801.07360].

[30] Q. Wu, G. Q. Zhang and F. Y. Wang, arXiv:2108.00581 [astro-ph.CO].

[31] X. Z. Er, Y. P. Yang and A. Rogers, Astrophys. J. 889, 158 (2020) arXiv:2001.02100.

[32] H. Zhou, Z. Li, H. Gao, Z. Huang and L. Huang, arXiv:2103.08510 [astro-ph.CO].

[33] S. Zhao, B. Liu, Z. Li and H. Gao, Astrophys. J. 916, no.2, 70 (2021).

[34] D. C. Qiang, H. K. Deng and H. Wei, Class. Quant. Grav. 37, 185022 (2020) arXiv:1902.03580].

[35] D. C. Qiang and H. Wei, JCAP 2004, 023 (2020) arXiv:2002.10189.

[36] D. C. Qiang and H. Wei, Phys. Rev. D 103, 083536 (2021) arXiv:2102.00579.

[37] K. E. Heintz et al., Astrophys. J. 903, 152 (2020) arXiv:2009.10747;

The up-to-date compilation of all known FRB host galaxies is available at https://frbhosts.org

[38] R. C. Zhang and B. Zhang, Astrophys. J. Lett. 924, no.1, L14 (2022) arXiv:2109.07558.

[39] W. Lu and A. L. Piro, Astrophys. J. 883, 40 (2019) arXiv:1903.00014.

[40] H. K. Vedantham, V. Ravi, G. Hallinan and R. Shannon, Astrophys. J. 830, 75 (2016) arXiv:1606.06795.

[41] W. Lu, P. Kumar and B. Zhang, Mon. Not. Roy. Astron. Soc. 498, 1397 (2020) arXiv:2005.06736.

[42] R. Luo et al., Mon. Not. Roy. Astron. Soc. 481, 2320 (2018) arXiv:1808.09929.

[43] R. Luo et al., Mon. Not. Roy. Astron. Soc. 494, 665 (2020) arXiv:2003.04848.

[44] F. Y. Wang and G. Q. Zhang, Astrophys. J. 882, 108 (2019) arXiv:1904.12408.

[45] K. Ioka, Astrophys. J. 598, L79 (2003) astro-ph/0309200].

[46] S. Inoue, Mon. Not. Roy. Astron. Soc. 348, 999 (2004) astro-ph/0309364.

[47] J. P. Macquart et al., Nature 581, no.7809, 391 (2020) arXiv:2005.13161.

[48] C. W. James et al., Mon. Not. Roy. Astron. Soc. 509, 4775 (2022) arXiv:2101.08005].

[49] C. W. James et al., Mon. Not. Roy. Astron. Soc. 510, L18 (2022) arXiv:2101.07998.

[50] P. Chawla et al. arXiv:2107.10858 [astro-ph.HE].

[51] B. C. Andersen et al., Nature 587, no. 7832, 54 (2020) arXiv:2005.10324.

[52] C. D. Bochenek et al., Nature 587, no. 7832, 59 (2020) arXiv:2005.10828.

[53] L. Lin et al., Nature 587, no. 7832, 63 (2020) arXiv:2005.11479.

[54] C. K. Li et al., Nat. Astron. 5, 378 (2021) arXiv:2005.11071.

[55] S. P. Tendulkar et al., Astrophys. J. Lett. 834, no.2, L7 (2017) arXiv:1701.01100.

[56] B. Marcote et al., Nature 577, no.7789, 190 (2020) arXiv:2001.02222.

[57] C. H. Niu et al., arXiv:2110.07418 [astro-ph.HE].

[58] M. Bhardwaj et al., Astrophys. J. Lett. 910, no.2, L18 (2021) arXiv:2103.01295.

[59] F. Kirsten et al., arXiv:2105.11445 [astro-ph.HE].

[60] K. Nimmo et al., arXiv:2105.11446 [astro-ph.HE].

[61] X. F. Cao, Y. W. Yu and X. Zhou, Astrophys. J. 858, no.2, 89 (2018) arXiv:1803.06266].

[62] N. Locatelli et al., Astron. Astrophys. 625, A109 (2019) arXiv:1811.10641.

[63] M. Amiri et al., Astrophys. J. Supp. 257, no.2, 59 (2021) arXiv:2106.04352.

The data for CHIME/FRB Catalog 1 in machine-readable format can be found via their public webpage at https://www.chime-frb.ca/catalog

[64] N. Aghanim et al., Astron. Astrophys. 641, A6 (2020) arXiv:1807.06209.

[65] H. Yüksel et al., Astrophys. J. Lett. 683, L5 (2008) arXiv:0804.4008. 
[66] P. Madau and M. Dickinson, Ann. Rev. Astron. Astrophys. 52, 415 (2014) arXiv:1403.0007.

[67] P. Madau and T. Fragos, Astrophys. J. 840, no.1, 39 (2017) arXiv:1606.07887.

[68] K. Dolag et al., Mon. Not. Roy. Astron. Soc. 451, no.4, 4277 (2015) arXiv:1412.4829.

[69] J. X. Prochaska and Y. Zheng, Mon. Not. Roy. Astron. Soc. 485, no.1, 648 (2019) arXiv:1901.11051.

[70] Z. X. Li et al., Mon. Not. Roy. Astron. Soc. 496, no.1, L28 (2020) arXiv:2004.08393.

[71] P. A. R. Ade et al., Astron. Astrophys. 594, A13 (2016) arXiv:1502.01589.

[72] B. Zhang, Astrophys. J. Lett. 867, no.2, L21 (2018) arXiv:1808.05277.

[73] J. M. Cordes and T. J. W. Lazio, astro-ph/0207156.

[74] J. M. Cordes and T. J. W. Lazio, astro-ph/0301598

[75] https://en.wikipedia.org/wiki/Kolmogorov-Smirnov_test

[76] https://docs.scipy.org/doc/scipy/reference/generated/scipy.stats.kstest.html

[77] M. D. Kistler et al., Astrophys. J. Lett. 673, L119 (2008) arXiv:0709.0381.

[78] F. J. Virgili et al., Mon. Not. Roy. Astron. Soc. 417, 3025 (2011) arXiv:1105.4650.

[79] F. Y. Wang and Z. G. Dai, Astrophys. J. Suppl. 213, 15 (2014) arXiv:1406.0568. 